\title{
Nonradiative resonance energy transfer directed from colloidal CdSe/ZnS quantum dots to epitaxial InGaN/GaN quantum wells for solar cells
}

\author{
Sedat Nizamoglu ${ }^{*, 1}$, Emre Sari', Jong-Hyeob Baek ${ }^{2}$, In-Hwan Lee ${ }^{3}$, and Hilmi Volkan Demir ${ }^{1,4}$ \\ ${ }^{1}$ Department of Electrical and Electronics Engineering, Department of Physics, and Institute of Materials Science \\ and Nanotechnology Bilkent University, Ankara 06800, Turkey \\ ${ }^{2}$ Korea Photonics Technology Institute, Gwangju 500-460, Korea \\ ${ }^{3}$ School of Advanced Materials Engineering, Research Center of Industrial Technology, Chonbuk National University, \\ Chonju 561-756, Korea \\ ${ }^{4}$ Microelectronics Division, School of Electrical and Electronics Engineering and Physics and Applied Physics Division, \\ School of Physical and Mathematical Sciences, Nanyang Technological University, Nanyang Avenue, Singapore 639798, Singapore
}

Received 27 April 2010, revised 27 May 2010, accepted 28 May 2010

Published online 4 June 2010

Keywords energy transfer, nanoscale, quantum dots, semiconductors, solar cells

${ }^{*}$ Corresponding author: e-mail sedatn@ee.bilkent.edu.tr

We report on Förster-type nonradiative resonance energy transfer (NRET) directed from colloidal quantum dots (QDs) to epitaxial quantum wells (QWs) with an efficiency of $69.6 \%$ at a rate of $1.527 \mathrm{~ns}^{-1}$ for potential application in III-nitride based photovoltaics. This hybrid exciton generation-collection system consists of chemically-synthesized cyan CdSe/ZnS core/shell QDs $\left(\lambda_{\mathrm{PL}}=490 \mathrm{~nm}\right)$ intimately integrated on epitaxially-grown green $\mathrm{InGaN} / \mathrm{GaN} \mathrm{QWs}$ $\left(\lambda_{\mathrm{PL}}=512 \mathrm{~nm}\right)$. To demonstrate directional NRET from donor QDs to acceptor QWs, we simultaneously show the decreased photoluminescence decay lifetime of dots and increased lifetime of wells in the hybrid dipole-dipole coupled system.
As a new material system for solar cell applications, III-nitrides - InGaN alloys in particular - offer significant benefits including direct band gap covering almost the entire visible spectrum, high electron and hole mobility, high drift velocity, and radiation resistance, making InGaN/GaN one of the most promising material systems for future photovoltaics [1-5]. One way of making solar cells using this material system is based on the approach of conventional photovoltaics using a $\mathrm{p}-\mathrm{i}-\mathrm{n}$ diode architecture that basically relies on generating excitons in $\mathrm{InGaN} / \mathrm{GaN}$ quantum wells (QWs) as a result of the optical absorption of the incident solar radiation and subsequently disassociating these excitons and separating their carriers in QWs with the help of the built-in electric field of the diode. However, the efficiency of this type of solar diodes is limited due to low light absorption by thin QW layers and undesired absorption of the incoming photons possibly in the top contact layer [6]. Alternatively, in this work, we inves- tigate and demonstrate an electrostatically coupled hybrid system of $\mathrm{CdSe} / \mathrm{ZnS}$ core/shell semiconductor quantum dots (QDs) with InGaN/GaN QWs in which colloidal QDs behave as the Förster-type nonradiative resonance energy transfer (NRET) donors to harvest incident light as an additional pathway and epitaxial QWs as the NRET acceptors to collect these harvested exciton energies. The advantage of such a hybrid system is that the enhancement of the effective absorption cross section of the quantum wells as a result of additional exciton harvesting in the quantum dots and the undesired electron-hole pairs generated in the top contact layer in a conventional solar cell can be reduced [7]. The feasibility of this type of NRET-based light harvesting into InGaN/GaN QWs offers new possibilities for InGaN material system important for future photovoltaics.

Recently such incoherently coupled hybrid nanostructures have been investigated in the literature. Agranovich 
et al. theoretically investigated the NRET concept between organic substances and inorganic quantum wells [8]. Rohrmoser et al. showed the temperature dependence of exciton transfer from a single InGaN quantum well donor to colloidal CdS quantum dot acceptors [9] and Heliotis et al. studied a temperature-dependent NRET analysis of a similar hybrid system [10]. Itskos et al. also investigated interactions between the organic polyfluorene thin films and single inorganic InGaN QWs with varying GaN cap layer thicknesses [11]. Achermann et al. showed nonradiative pumping of QDs with exciton transfer from QWs [12]. For future solid state lighting devices, our group also previously demonstrated white light generating nonradiative energy transfer from epitaxial QWs to proximal colloidal QDs [13]. Furthermore, NRET has also been previously demonstrated from quantum dots to adjacent quantum channels made of InGaAs quantum wells grown on GaAs and to nanowires made of silicon $[14,15]$. Also, more recently, photocurrent enhancement has been shown using hybrid nanocrystal quantum-dot $\mathrm{p}-\mathrm{i}-\mathrm{n}$ photovoltaic devices that include $\mathrm{GaAs} / \mathrm{AlGaAs}$ quantum wells in patterned vertical arrays [16]. Different from the previous studies, in this Letter we present nonradiative resonance energy transfer from $\mathrm{CdSe} / \mathrm{ZnS}$ core/shell colloidal quantum dots to InGaN/GaN epitaxial quantum wells important for future III-nitrides based photovoltaics. We experimentally demonstrate this QD-to-QW directed NRET with an efficiency level of $69.6 \%$ at a rate of $1.527 \mathrm{~ns}^{-1}$. To prove this directional process of NRET we show both the lifetime decrease of quantum dots (as the donors) and the lifetime increase of quantum wells (as the acceptors), simultaneously observed in the hybrid exciton transfer system. This means that it is possible in principle to separate the associated tasks of exciton generation at highly disordered, strongly absorbing colloidal QDs to transfer their excitonic energy to exciton collection centers of highly ordered epitaxial InGaN/GaN QWs embedded in a diode architecture to efficiently disassociate the collected excitons and efficiently conduct their separated charges. This may enable highly efficient excitonic optoelectronic devices of IIInitrides including excitonic InGaN/GaN solar cells.

As nanostructures to be investigated in our hybrid system we use colloidal cyan-emitting $\mathrm{CdSe} / \mathrm{ZnS}$ core/shell quantum dots and green-emitting epitaxial InGaN/GaN multi-quantum wells. The photoluminescence emission peak of these QDs (acquired from Evident Technologies) is at $490 \mathrm{~nm}$ in toluene, and they have a concentration of $15.85 \mathrm{nmol} / \mathrm{ml}$ with a size dispersion of $<5 \%$. To prepare the InGaN/GaN epi-wafer we grow $3.5 \mu \mathrm{m}$ n-doped GaN on top of the polished sapphire substrate. Afterwards we continue with $5 \mathrm{InGaN} / \mathrm{GaN}$ well/barrier layers and finally finish our epitaxial structure with a few monolayers of undoped GaN capping layer. According to the X-ray diffraction measurement (XRD) shown in the inset of Fig. 1, the well and barrier thicknesses are around $89 \mathrm{~nm}$, with an indium mole fraction of $72 \%$ in the well, resulting in a photoluminescence peak around $512 \mathrm{~nm}$. The QWs are epi-

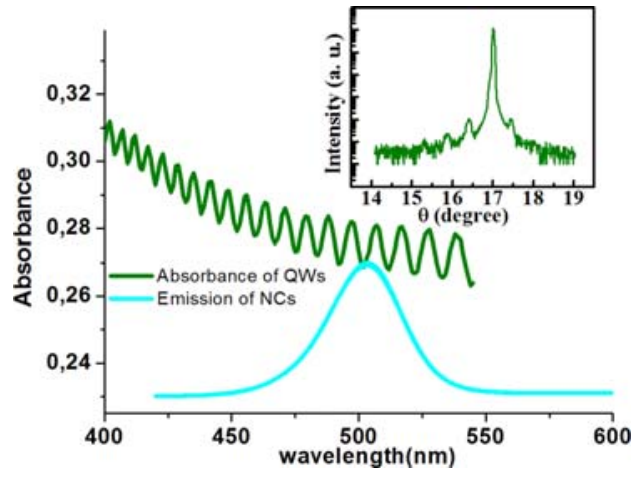

Figure 1 (online colour at: www.pss-rapid.com) Photoluminescence of $\mathrm{CdSe} / \mathrm{ZnS}$ core/shell quantum dots in film (cyan solid line) and absorbance of InGaN/GaN quantum wells (green line) along with the X-ray diffraction measurement (inset).

taxially grown using $2 \mathrm{~nm}$ thick well and $16 \mathrm{~nm}$ think barrier of an uncoupled multiple quantum well structure design. The center to center distance is equal to $\sim 3.8 \mathrm{~nm}$ ( $\sim 1 \mathrm{~nm}$ QW center to GaN capping layer $+1 \mathrm{~nm} \mathrm{GaN} \mathrm{cap-}$ ping layer thickness $+1.8 \mathrm{~nm}$ ligand and QD radius) leading to efficient NRET. Since these are uncoupled QWs (due to their thick barrier layer), farther QWs except for the nearest one do not contribute to the NRET process.

For efficient exciton migration from donor molecules to acceptor molecules, the donors are required to possess a high level of quantum efficiency. In our hybrid system our $\mathrm{CdSe} / \mathrm{ZnS}$ core/shell quantum dot donors exhibit a relatively high quantum efficiency (e.g., $>50 \%$ in solution) that leads to reasonably efficient NRET. Furthermore, the absorption of the acceptor and emission of the donor should feature a large spectral overlap. As also shown in Fig. 1, the absorption of QWs and emission of QDs overlap sufficiently well for strong dipole-dipole coupling in the hybrid system. For QD film formation, QDs are deposited by drop-casting on top of the substrate. Finally, excess QDs are removed by washing off and those that are attached to the substrate remain. For the reference sample, QDs are integrated on top of the quartz substrate with the same amount as in the hybrid system case.

For time-resolved investigations we use a diode laser as the excitation source emitting at $375 \mathrm{~nm}$ and as the detector we utilize a photon multiplier tube (PMT) with a calibrated time sampling rate of 64 ps between two adjacent data points. For the numerical analysis of the experimental data, we deconvolute the instrumental response function (IRF) as shown in the inset of Fig. 2. (Since in our system we have an instrument response function with a finite line width in time, the actual response seen in the figures is in fact the convoluted response with the IRF, which we should take into account in our calculations and make the fitting accordingly. Thus, the curves in the log-scale do not seem as perfect straight lines.) Starting with the analysis of the time-resolved data in Fig. 2, the photoluminescence decay curve of the hybrid sample at $460 \mathrm{~nm}$ drops faster with respect to the only cyan-emitting QDs. 


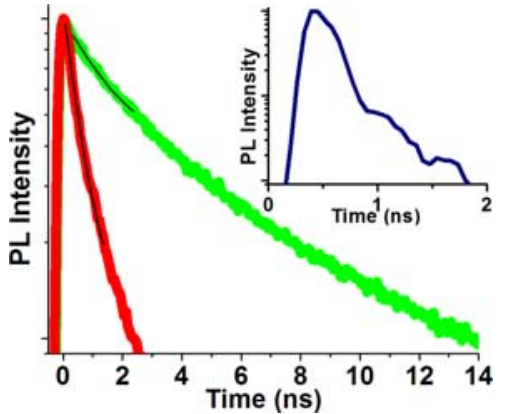

Figure 2 (online colour at: www.pss-rapid.com) Time-resolved spectroscopy of our hybrid system (red solid line) compared against only quantum dots (green solid line), both measured at the emission wavelength of $460 \mathrm{~nm}$. The solid black lines are the fitted curves. Inset shows the response decay of the excitation laser.

Here this is expectedly because the excitons generated in donor quantum dots are transferred to and collected at the acceptor quantum wells in the hybrid structure. By using a single exponential model we fit our donor decays shown in Fig. 2 to evaluate the energy transfer efficiency. The rate of only QDs (i.e., $k_{\mathrm{QD}}$ ) corresponds to $0.665 \mathrm{~ns}^{-1}$ and the rate of the hybrid system $\mathrm{QD}+\mathrm{QW}$ (i.e., $k_{\mathrm{QD}+\mathrm{QW}}$ ) is equal to $2.192 \mathrm{~ns}^{-1}$. By using these rates, we calculate the NRET rate $k_{\mathrm{NRET}}=k_{\mathrm{QD}+\mathrm{QW}}-k_{\mathrm{QD}}=1.527 \mathrm{~ns}^{-1}$. As a result, the hybrid system accomplishes a NRET efficiency of $69.6 \%$ $\left(\eta=k_{\mathrm{NRET}} /\left(k_{\mathrm{NRET}}+k_{\mathrm{QD}}\right)=k_{\mathrm{NRET}} / k_{\mathrm{QD}+\mathrm{QW}}\right)$, meaning that most of the excitons generated in QDs are transferred to nearby QWs. One possible question here is whether this energy transfer is a Förster-type or a Dexter-type process. Since in our core/shell heteronanocrystals $\mathrm{ZnS}$ barriers provide full electronic isolation and completely prevent tunneling of the electron and hole wavefunctions, this cannot be due to the Dexter-type transfer. Thus, this transfer needs to take place as a result of nonradiative energy transfer from quantum dots to quantum wells.

To verify NRET we further investigate the timeresolved spectroscopy of our hybrid system at the acceptor emission on the transient QW dynamics (as shown in Fig. 1, the emission of the QDs is negligible at $540 \mathrm{~nm}$ ). In Fig. 3 the time-resolved photoluminescence of the hybrid sample at $540 \mathrm{~nm}$ shows a slower decay with respect to the only green-emitting QWs because these acceptor wells are fed with the transferred energy from the donor dots in the hybrid structure with respect to the only green-emitting wells. To observe NRET in the acceptor emission, the fluorescence dynamics of the hybrid system is subtracted from the dynamics of the only quantum wells and the difference curve is shown in the inset of Fig. 3. Here this behaviour is due to the carrier injection from the QDs to the QWs [17] and thus, we can conclusively state that this process is a NRET from QDs to QWs. As a remark, in the inset of Fig. 3 the lifetime increase for the hybrid sample with respect to the only green-emitting QWs is not as pronounced because only the top QW is nonradiatively pumped and the other remaining four QWs are radiatively pumped.

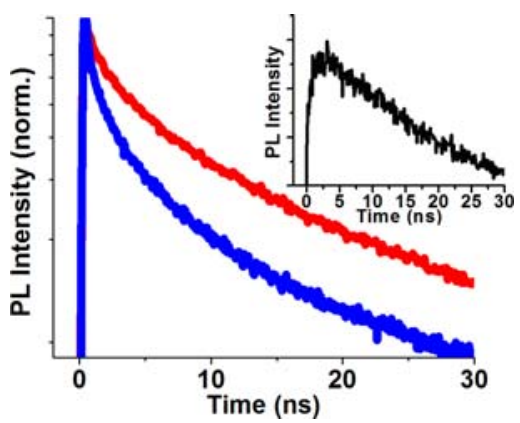

Figure 3 (online colour at: www.pss-rapid.com) Time-resolved spectroscopy of our hybrid system (red solid line) compared against only quantum wells (blue solid line) both measured at the emission wavelength of $540 \mathrm{~nm}$. Inset shows the difference between the decay dynamics of hybrid system and only quantum wells (black solid line).

In conclusion, we presented nonradiative Förster-type resonance energy transfer from colloidal quantum dots to epitaxial quantum wells for III-nitride based photovoltaic applications. Because of NRET directed from QDs to QWs, we observed the lifetime decrease in quantum dots and lifetime increase in quantum wells. This scheme achieved a NRET efficiency of approximately $69.6 \%$ and a NRET rate of $1.527 \mathrm{~ns}^{-1}$. In such a device structure the efficiency limitations due to the absorption of the incoming radiation by the top contact layer and low light absorption by the thin QW layer can be reduced. As a next step, making electrical contacts to this type of hybrid system and studying NRET enhanced InGaN solar cells are underway.

Acknowledgements We acknowledge the financial support by ESF-EURYI (European Young Investigator Award program), EU FP7 Nanophotonics4Energy NoE, ESF COST MP0701 and MP0702, and TUBITAK under the Project Nos. EEEAG 106E020, 107E088, 107E297, 109E002, and 109E004. H.V.D. acknowledges additional support from the Turkish National Academy of Sciences Distinguished Young Scientist Award (TUBA GEBIP).

\section{References}

[1] O. Jani et al., Appl. Phys. Lett. 91, 132117 (2007).

[2] J. Wu et al., J. Appl. Phys. 94, 6477 (2003).

[3] Y. Nanishi et al., Jpn. J. Appl. Phys. 42, 2549 (2003).

[4] M. Vazquez et al., Progr. Photovoltaics 15, 477 (2007).

[5] C. J. Neufeld et al., Appl. Phys. Lett. 93, 143502 (2008).

[6] R. Dahal et al., Appl. Phys. Lett. 94, 063505 (2009).

[7] M. Achermann et al., J. Phys. Chem. B 107, 13782 (2003).

[8] V. Agranovich et al., Solid State Comm. 92, 295 (1994).

[9] S. Rohrmoser et al., Appl. Phys. Lett. 91, 092126 (2007).

[10] G. Heliotis et al., Adv. Mater. 18, 334 (2006).

[11] G. Itskos et al., Phys. Rev. B 76, 035344 (2007).

[12] M. Achermann et al., Nature 429, 642 (2004).

[13] S. Nizamoglu et al., New J. Phys. 10, 123001 (2008).

[14] S. Lu and A. Madhukar, Nano Lett. 7, 3443 (2007).

[15] S. Lu et al., Nano Lett. 9, 12 (2009).

[16] S. Chanyawadee et al., Phys. Rev. Lett. 102, 077402 (2009).

[17] S. Chanyawadee et al., Adv. Mater. 22, 602 (2010). 